\title{
Aprendizaje colaborativo y tecnologías de información y comunicación para el perfil global de docentes y estudiantes universitarios ${ }^{1}$
}

\section{Collaborative learning and information and communication technologies for the global profile of university teachers and students}

\author{
María Claudia Aponte ${ }^{2}$ (D) y Orlando Brea ${ }^{3}$ (iD
}

Para citar este artículo: Aponte, M. C. y Brea, O. (2019). Aprendizaje colaborativo y tecnologías de información y comunicación para el perfil global de docentes y estudiantes universitarios. Revista ObIES, 3, 88-100.

Recibido: 13-agosto-2019 / Aceptado: 11-noviembre-2019

\section{Resumen}

Investigadores de la internacionalización de la educación superior y del currículo, asociaciones universitarias de facultades y escuelas, organismos internacionales, investigadores de la pedagogía y del currículo han trabajado en el establecimiento de las características y competencias para la formación de perfiles internacionales para los profesionales de hoy y del futuro. El aprendizaje colaborativo y la aplicación de las TIC al proceso de aprendizaje son unas de las competencias más mencionadas en las propuestas sobre el perfil global del estudiante y del profesor. Pero, ¿qué tan dispuestos están los profesores y los estudiantes para desarrollar estas competencias? Este artículo presenta los resultados de un proyecto de innovación que incorporó en procesos formativos en diferentes grupos de estudiantes y de profesores, el aprendizaje colaborativo y las TIC. La hipótesis del trabajo planteó que para los docentes universitarios la inclusión de estas competencias en su perfil generaría una resistencia, mientras que para los estudiantes no sería así. Los resultados mostraron que el aprendizaje colaborativo se aplica ocasionalmente en ambos grupos, los docentes y estudiantes utilizan frecuentemente las TIC en sus procesos de aprendizaje, y para los docentes el uso de TIC y el aprendizaje colaborativo resultan más difíciles de aplicar, pero cuando deben lo hacen sin mayores resistencias, aunque de manera básica.

Palabras clave: educación superior; perfil global; competencias globales; aprendizaje colaborativo; tecnologías de información y comunicación.

\begin{abstract}
Researchers on the internationalization of higher education and the curriculum, university associations of faculties and schools, international organizations, researchers on pedagogy and the curriculum have worked on establishing the characteristics and competencies for the formation of international profiles for the professionals of today and the future. Collaborative learning and the application of ICTs to the learning process are some of the competencies most mentioned in the proposals on the global student and teacher profile. But
\end{abstract}

1 Proyecto de innovación "Resistencias en la implementación de nuevas didácticas para el desarrollo de competencias globales", realizado para la certificación de los autores en el Programa de Certificación de Educador Internacional de Ingeniería (IGIP).

2 Consultora en Educación Internacional, Colombia. Correo electrónico: apontemac@gmail.com

3 Universidad Tecnológica Nacional, Argentina. Correo electrónico: orlando.brea@gmail.com 
how willing are teachers and students to develop these competences? This article presents the results of an innovation project that incorporated collaborative learning and ICTs into training processes in different groups of students and teachers. The working hypothesis was that for university teachers the inclusion of these competences in their profile would generate a resistance, while for students it would not be so. The results showed that collaborative learning is occasionally applied to both groups, teachers and students frequently use ICTs in their learning processes, and for teachers the use of ICTs and collaborative learning is more difficult to apply, but when it must be done, it is done without much resistance, albeit in a basic way.

Keywords: higher education; global profile; global skills; collaborative learning; information and communication technologies.

\section{Introducción}

La internacionalización del currículo universitario debe generar impactos sobre la formación de los estudiantes, especialmente en su perfil internacional. Para esto, el profesor afronta el desafío de integrar las dimensiones internacionales, interculturales e interdisciplinarias en su estrategia de aula, en los contenidos de los cursos o asignaturas, en el método didáctico y en la evaluación, así como en el desarrollo de las competencias globales asociadas al perfil internacional. La Organización para la Cooperación y el Desarrollo Económicos (OCDE, 1998) considera que un currículo tiene orientación internacional en contenido o forma cuando este es capaz de preparar tanto estudiantes nacionales como extranjeros para realizarse (profesionalmente/socialmente) en un contexto internacional y multicultural. Esta formación se conoce con los términos "perfil global", "profesional global" o "perfil internacional".

\section{El perfil global de egreso de un universitario}

Es cierto que una experiencia de movilidad internacional impacta la vida de un estudiante y su manera de ver el mundo, pero la gran mayoría de estudiantes no tendrán acceso a esta experiencia, por lo que es necesario que el profesor ofrezca oportunidades y escenarios de formación de competencias globales en el contexto del aula. Aponte, Jordan y Agi (2017) advierten que las brechas en la formación de profesionales globales se seguirán abriendo si los procesos de internacionalización se continúan viendo como actividades aisladas de los entornos globales y si los docentes universitarios no dirigen sus esfuerzos a la formación de profesionales globales desde los ambientes de aprendizaje. Investigadores de la internacionalización de la educación superior y del currículo, asociaciones universitarias de facultades y escuelas, organismos internacionales, investigadores de la pedagogía y del currículo vienen proponiendo en los últimos años perfiles internacionales para los profesionales de hoy y del futuro. Lo que hace a un profesional global es el desarrollo de las competencias globales. La OCDE (2018) define una competencia global como la capacidad de analizar de manera critica y desde múltiples perspectivas las cuestiones globales e interculturales, con el objetivo de comprender cómo las diferencias afectan las percepciones, los juicios y las ideas de sí mismo y de los demás para entablar interacciones abiertas, apropiadas y efectivas con personas de diferentes orígenes sobre la base del respeto compartido de la dignidad humana. Este organismo, en el documento "Working Paper 21st Century Skills and Competences for New Millennium Learners in OECD Countries", establece tres grupos de competencias para el profesional del siglo XXI:

- Uso interactivo de las herramientas digitales, lo cual incluye habilidades para buscar, seleccionar, evaluar y organizar información en un mar de datos, así como reestructurar y modelar información para crear nuevo conocimiento. 
- Interacción entre grupos heterogéneos, lo que conlleva habilidades para la comunicación y la colaboración e interacción virtual.

- Actuación de forma autónoma y ética, con responsabilidad social y conciencia de los impactos y transformaciones sociales.

Aponte (2018) propone en el documento "Competencias del perfil global" tener en cuenta para el establecimiento de un perfil global de egreso de la educación superior las siguientes competencias del proyecto Alfa Tünning para América Latina: 1) responsabilidad social y compromiso ciudadano, 2) capacidad de comunicación en un segundo idioma, 3) capacidad para actuar en nuevas situaciones, 4) capacidad de trabajo en equipo, 5) valoración y respeto por la diversidad y multiculturalidad y 6) habilidad para trabajar en contextos internacionales.

Aponte y Jordán (2017) plantean que no hay un solo perfil de los profesionales globales; existen propuestas sobre perfiles generales y también específicos para las profesiones. Lo ideal es que el perfil se estructure a partir de unas competencias o unas capacidades profesionales de orden global y unas capacidades transversales globales. Estas últimas se denominan también habilidades para el siglo XXI, o habilidades blandas, y se constituyen hoy en día como una prioridad para la educación universitaria en el mundo. Organismos internacionales como el Foro Económico Mundial, y otros organismos del orden nacional en estudios sobre puestos de trabajos, evidencian de manera reiterada la necesidad de que los postulantes tengan las Ilamadas habilidades blandas o competencias transversales como lo son: la capacidad de trabajar en equipo, de sugerir mejoras a los procesos (comunicación e involucración), habilidades para la resolución de problemas y el uso de herramientas de comunicación digitales.

En cuanto a las competencias disciplinares del orden global, diversas disciplinas ya han definido qué se requiere para formar a sus egresados como profesionales globales. Es el caso de la ingeniería que se preocupa por la calidad de la formación de nuevas generaciones de ingenieros, para que sean capaces de "entender el impacto de las soluciones de ingeniería en un contexto global, económico, medioambiental, y social" (Felder y Brent, 2004). En esta y otras disciplinas se reiteran la necesidad que los profesionales del futuro tengan una fuerte formación en competencias disciplinares que les permitan desempeñarse en contextos globales, pero que haya un total equilibrio con el desarrollo de competencias transversales globales o habilidades para los nuevos tiempos.

\section{El proyecto de innovación}

El proyecto de innovación "Resistencias en la implementación de nuevas didácticas para el desarrollo de competencias globales", se realizó por los autores del artículo (un profesor universitario argentino y una profesora en educación internacional colombiana), en el marco del Programa de Certificación de Educador Internacional de Ingeniería (IGIP). Se trata de un programa acreditado internacionalmente de formación de profesores universitarios de ingeniería y disciplinas afines, como profesores globales, en el cual los autores tuvieron que proponer un proyecto de innovación aplicado a su contexto: el profesor argentino hizo la innovación con su grupo de estudiantes de pregrado y la profesora colombiana con su grupo de estudiantes, quienes eran profesores universitarios.

El propósito del proyecto de innovación fue desarrollar dos competencias del perfil global en dos grupos de estudiantes y dos grupos de profesores de la educación superior a través de la aplicación de didácticas centradas en el estudiante. La hipótesis de la innovación planteó que para los docentes universitarios la inclusión de estas dos competencias - aprendizaje colaborativo y manejo de $\mathrm{TIC}$ - en su proceso formativo generaría resistencia en la implementación, mientras que para los estudiantes no lo haría. 
El curso de "Competencias del perfil global" impartido por la docente colombiana a 44 docentes de la educación superior en Colombia fue de 40 horas presenciales con trabajo independiente. Para el caso de los estudiantes del profesor argentino, se desarrolló el curso "Experto universitario en PHP y MySQL" impartido a seis estudiantes de pregrado de Argentina tuvo una duración de 180 horas presenciales, mientras que el curso "Professional webmaster" fue impartido a 20 estudiantes de pregrado con un trabajo presencial de 144 horas.

Durante los cursos tomados por los profesores en Colombia y los estudiantes en Argentina se aplicaron los mismos ejercicios para el uso de las TIC, de acuerdo con las pautas de "aprendiz empoderado" y "comunicador creativo" propuestos por la Sociedad Internacional de Tecnología en Educación (Iste) para estudiantes. Esos son: 1) búsqueda en internet, 2) correo electrónico, 3) plataforma virtual de apoyo al curso, 4) Whatsapp, 5) Google Drive, 6) Trello y otros. Para la competencia del aprendizaje colaborativo se aplicaron en los grupos de docentes de Colombia y de estudiantes de Argentina las mismas técnicas para la dinámica de los equipos de trabajo que se constituyeron al inicio de cada curso de formación con el objetivo de desarrollar ejercicios colaborativos: definición de un nombre y un logo del equipo, acuerdo sobre unos valores que regirían el trabajo colaborativo, establecimiento de unas normas de autorregulación para la resolución de conflictos durante el curso, definición de unas pautas para garantizar el rendimiento y el cumplimiento de las responsabilidades individuales, compromiso de unos papeles básicos que debían rotarse (como mínimo la coordinación de la dinámica del trabajo colaborativo y la relatoría de la actividad realizada), así como la construcción de una rúbrica de evaluación del trabajo colaborativo (Aponte, 2018a).

\section{Aprendizaje colaborativo y TIC para el perfil global}

El proyecto de innovación seleccionó dos competencias, nombradas en múltiples trabajos de la literatura consultada como competencias básicas para el perfil global de los estudiantes universitarios. Estas competencias de aprendizaje colaborativo y TIC deben ser desarrolladas tanto por los docentes como los estudiantes. El proyecto de innovación tuvo una base conceptual para el desarrollo de estas competencias.

El aprendizaje colaborativo se define como el:

[...] conjunto de métodos de instrucción o entrenamiento para uso en grupos, así como de estrategias para propiciar el desarrollo de habilidades mixtas (aprendizaje, desarrollo personal y social). En el aprendizaje cooperativo cada uno es responsable de su propio aprendizaje, así como el de los restantes miembros del grupo (Johnson, 1993).. (Begué et al., 2012, p. 34).

Por otro lado, el aprendizaje colaborativo:

[...] consiste en la habilidad de desarrollar nuevas ideas entre los miembros de un grupo para lograr un aprendizaje común. Para la construcción conjunta del conocimiento, el aprendizaje colaborativo exige nuevos roles tanto de estudiantes como de los profesores, siempre en un ambiente de respeto. (Aponte, 2018a, p. 2)

El aprendizaje colaborativo deber ser aprendido, pues tiene una serie de criterios y técnicas para su éxito. Normalmente, en el ámbito educativo universitario se da por sentado que tanto docentes como estudiantes saben aprender y trabajar de manera colaborativa, pero esto no es tan natural. Lillo (2013) recalca que este aprendizaje implica un cambio cultural, pues no se da de forma natural al dar una instrucción a un grupo de personas, por 
lo que debe existir una intención, unos objetivos de formación comunes para el equipo de trabajo y unas pautas y unas técnicas previamente planeadas. Estos criterios y técnicas se relacionan con la formalización del trabajo de los equipos y el establecimiento de pautas de autorregulación.

[...] el aprendizaje colaborativo no tiene una forma única, y por lo mismo, es flexible la forma de ser aplicado, permitiendo que el docente realice cambios durante el proceso. Sin embargo, resulta primordial un buen diseño y planificación previa a la aplicación de la metodología, que considere todos los aspectos relevantes para facilitar el aprendizaje colaborativo, que además dé paso a que se generen productos o resultados que puedan tener una trascendencia útil y constituyan evidencias de un aprendizaje significativo. (Lillo, 2013a, p. 138)

La otra competencia definida para el proyecto de innovación fue la de aplicación y uso de TIC, la cual está lo bastante bien estudiada en el mundo. Se denominan TIC: "al conjunto convergente de tecnologías que permiten la adquisición, producción, almacenamiento, tratamiento, comunicación, registro, acceso y presentación de datos, información y contenidos (en forma alfanumérica, imágenes, videos, sonidos, aromas, otros)" (Benvenuto, 2003, p. 113).

La Iste determina siete pautas para la incorporación de las TIC al aprendizaje y a la vida, por parte de un estudiante: aprendiz empoderado, ciudadano digital, constructor de conocimiento, diseñador innovador, pensador computacional, comunicador creativo y colaborador global.

Los estándares están diseñados para ser usados por educadores en todo el currículo con estudiantes de todas las edades, con el objetivo de cultivar estas habilidades a lo largo de toda la carrera académica. Tanto los estudiantes como los profesores serán responsables de lograr las habilidades tecnológicas fundamentales para aplicar plenamente los estándares. (Iste, 2016)
Para el caso de los docentes, las siete pautas definidas por la Iste son: 1) aprendices, 2) líderes, 3) ciudadanos, 4) colaboradores, 5) diseñadores, 6) facilitadores y 7) analistas. Estas no se trabajaron en el proyecto de innovación, pues los profesores participantes en el grupo de formación colombiano desempeñaron la función de estudiantes.

\section{Instrumentos del proyecto de innovación}

El proyecto de innovación desarrollado entre los años 2018 y 2019 tuvo como objetivo identificar en un conjunto de docentes en Colombia y un conjunto de estudiantes universitarios en Argentina la apertura para incorporar dos competencias globales. En el caso de los profesores, el proyecto de innovación se aplicó a dos grupos docentes de diversas disciplinas de la educación superior en Colombia. Por otro lado, el proyecto se aplicó a dos grupos de estudiantes de pregrado de la Facultad Regional Buenos Aires de Argentina.

Al finalizar los cursos se utilizaron en todos los grupos que participaron en el proyecto de innovación unos instrumentos previamente diseñados y validados para identificar qué tanto aceptaron las técnicas de aprendizaje colaborativo y TIC. Todos los participantes en los cursos de formación respondieron el instrumento, para un total de 70 personas, de los cuales 44 eran docentes universitarios y 26 alumnos de pregrado.

Se analizaron diferentes instrumentos para medir los resultados, tales como encuestas, entrevistas, observación, entre los que se seleccionó la realización de encuestas cerradas. Se eligió esta metodología por ser el instrumento que mejor se adaptaba a las dinámicas de los cursos y menor subjetividad podía tener del ejecutor, ya que el instrumento debía poder ser aplicado a diferentes grupos poblacionales (estudiantes en Argentina y profesores universitarios en Colombia) en contextos diversos.

Los instrumentos aplicados fueron los siguientes: 
Tabla 1. Instrumento de identificación de la aceptación del aprendizaje colaborativo

\begin{tabular}{|c|c|c|c|c|c|}
\hline $\begin{array}{l}\text { Antes del curso trabajé en equipos cola- } \\
\text { borativos con reglas de juego previamente } \\
\text { definidas. }\end{array}$ & $\begin{array}{l}\text { Muy frecuente- } \\
\text { mente }\end{array}$ & Frecuentemente & Ocasionalmente & Raramente & Nunca \\
\hline $\begin{array}{l}\text { El trabajo colaborativo durante el curso me } \\
\text { resultó: }\end{array}$ & Muy fácil & Fácil & Ni fácil ni difícil & Difícil & Muy difícil \\
\hline $\begin{array}{l}\text { En los trabajos colaborativos del curso, es- } \\
\text { cuché con atención las ideas y las opinio- } \\
\text { nes de los compañeros. }\end{array}$ & $\begin{array}{l}\text { Muy frecuente- } \\
\text { mente }\end{array}$ & Frecuentemente & Ocasionalmente & Raramente & Nunca \\
\hline $\begin{array}{l}\text { En los trabajos colaborativos del curso, res- } \\
\text { peté las pautas de mi grupo de trabajo. }\end{array}$ & $\begin{array}{l}\text { Muy frecuente- } \\
\text { mente }\end{array}$ & Frecuentemente & Ocasionalmente & Raramente & Nunca \\
\hline $\begin{array}{l}\text { En los trabajos colaborativos del curso, par- } \\
\text { ticipé de manera activa. }\end{array}$ & $\begin{array}{l}\text { Muy frecuente- } \\
\text { mente }\end{array}$ & Frecuentemente & Ocasionalmente & Raramente & Nunca \\
\hline $\begin{array}{l}\text { En los trabajos colaborativos del curso, } \\
\text { aproveché las ideas de los compañeros. }\end{array}$ & $\begin{array}{l}\text { Muy frecuente- } \\
\text { mente }\end{array}$ & Frecuentemente & Ocasionalmente & Raramente & Nunca \\
\hline $\begin{array}{l}\text { En los trabajos colaborativos del curso, le } \\
\text { ayudé a los compañeros en las tareas que } \\
\text { ellos no podían cumplir. }\end{array}$ & $\begin{array}{l}\text { Muy frecuente- } \\
\text { mente }\end{array}$ & Frecuentemente & Ocasionalmente & Raramente & Nunca \\
\hline $\begin{array}{l}\text { En los trabajos colaborativos del curso, me } \\
\text { sentí parte del grupo. }\end{array}$ & $\begin{array}{l}\text { Muy frecuente- } \\
\text { mente }\end{array}$ & Frecuentemente & Ocasionalmente & Raramente & Nunca \\
\hline $\begin{array}{l}\text { En los trabajos colaborativos del curso, las } \\
\text { cargas de trabajo fueron equitativas. }\end{array}$ & $\begin{array}{l}\text { Totalmente de } \\
\text { acuerdo }\end{array}$ & De acuerdo & Indeciso & $\begin{array}{l}\text { En des- } \\
\text { acuerdo }\end{array}$ & $\begin{array}{l}\text { Totalmente en } \\
\text { desacuerdo }\end{array}$ \\
\hline $\begin{array}{l}\text { Al realizar la coevaluación del trabajo cola- } \\
\text { borativo de mis compañeros de grupo, me } \\
\text { sentí bien. }\end{array}$ & $\begin{array}{l}\text { Totalmente de } \\
\text { acuerdo }\end{array}$ & De acuerdo & Indeciso & $\begin{array}{l}\text { En des- } \\
\text { acuerdo }\end{array}$ & $\begin{array}{l}\text { Totalmente en } \\
\text { desacuerdo }\end{array}$ \\
\hline
\end{tabular}

Fuente: elaboración propia, adaptado de Aponte (2018a).

Tabla 2. Instrumento de identificación de la aceptación del uso de las TIC

\begin{tabular}{|c|c|c|c|c|c|}
\hline \multicolumn{6}{|c|}{ 2- Aplicación de TIC } \\
\hline $\begin{array}{l}\text { Antes del curso aplicaba TIC al pro- } \\
\text { ceso de enseñanza-aprendizaje. }\end{array}$ & $\begin{array}{l}\text { Muy frecuente- } \\
\text { mente }\end{array}$ & Frecuentemente & Ocasionalmente & Raramente & Nunca \\
\hline $\begin{array}{l}\text { La aplicación de TIC a las actividades } \\
\text { del curso me resultó: }\end{array}$ & Muy fácil & Fácil & Ni fácil ni difícil & Difícil & Muy difícil \\
\hline $\begin{array}{l}\text { Para comunicarme con mi grupo } \\
\text { durante el curso usé: (puede marcar } \\
\text { más de una opción) }\end{array}$ & Correo & Skype & Whatsapp & Trello & Otras \\
\hline $\begin{array}{l}\text { Para realizar los trabajos en grupo } \\
\text { durante el curso usé: (puede marcar } \\
\text { más de una opción) }\end{array}$ & Google Drive & Dropbox & Docs & Otra & Ninguna \\
\hline $\begin{array}{l}\text { Para elaborar y presentar mis traba- } \\
\text { jos y los trabajos de grupo durante el } \\
\text { curso usé: (puede marcar más de una } \\
\text { opción) }\end{array}$ & Mind manager & Power point & Video & Otra & Ninguna \\
\hline $\begin{array}{l}\text { Para realizar los trabajos durante el } \\
\text { curso usé: (puede marcar más de una } \\
\text { opción) }\end{array}$ & Google & $\begin{array}{l}\text { Google acadé- } \\
\text { mico }\end{array}$ & Base de datos & $\begin{array}{l}\text { Revista } \\
\text { indexada }\end{array}$ & Otra \\
\hline
\end{tabular}

Fuente: elaboración propia adaptada de Barroso (2007). 
Los ítems que constituyen el instrumento de aprendizaje colaborativo ascienden a ocho y los de las TIC a seis, con opciones de respuesta en una escala de Likert, en la mayoría de preguntas, y en otras de opción múltiple cerradas.

\section{Los resultados del proyecto de innovación}

Una vez aplicados los dos instrumentos a los grupos de docentes y de estudiantes, para determinar la aceptación de las técnicas de aprendizaje colaborativo y las TIC (lo cual se realizó en la última sesión del curso), se obtuvieron los siguientes resultados:

En los grupos de docentes universitarios se encontró que antes del curso el 77 \% no había usado técnicas de trabajo colaborativo de manera habitual; que durante el proceso formativo la mayoría de docentes aceptó y aplicó las técnicas al iniciar el curso, aunque el 25 \% reconoció que no le resultó fácil; el $23 \%$ no colaboró con el aprendizaje de sus homólogos; el 20 \% sintió que las cargas de trabajo no fueron equitativas; y el $25 \%$ no se sintió bien evaluando el compromiso y trabajo de sus compañeros.

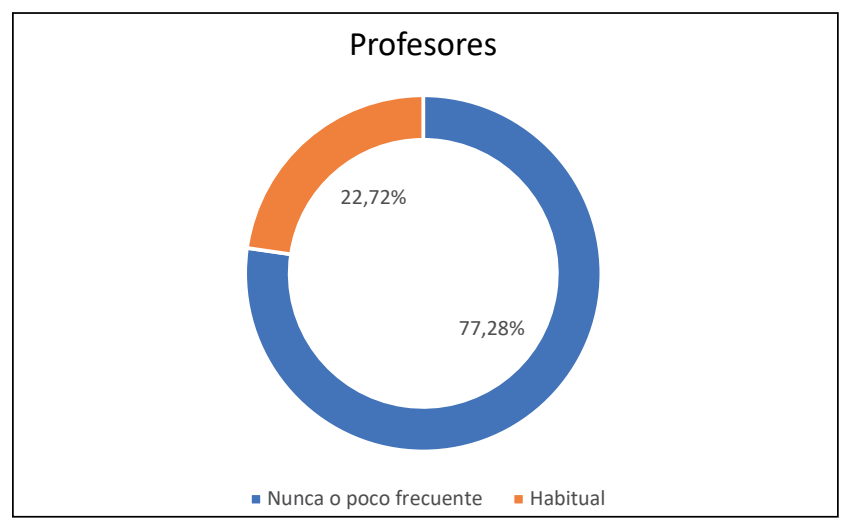

Figura 1. Trabajo en equipo previo al curso de profesores.
En los grupos de estudiantes universitarios se encontró que antes del curso el 90 \% no había utilizado técnicas planeadas de trabajo colaborativo; que durante el proceso formativo la mayoría de estudiantes aceptó y aplicó las técnicas al iniciar el curso; aunque el $10 \%$ aceptó que no le resultó fácil escuchar a sus compañeros ni sentirse parte del grupo. El 100 \% reconoció que se sintió bien evaluando el compromiso y trabajo de sus compañeros.

\section{Trabajo en equipo previo al curso}

Previo al curso, los profesores utilizaban de manera más habitual el trabajo en equipo que los alumnos. Ninguno de los grupos hace uso del trabajo en equipo de manera constante, lo cual permite diferenciar el objetivo de obtener capacidades para un perfil global por medio del trabajo en equipo y la realidad del trabajo diario; esto, teniendo presente el reto de que el trabajo colaborativo debe ser una "filosofía de interacción y una forma personal de trabajo" (Maldonado, 2007, p. 269).

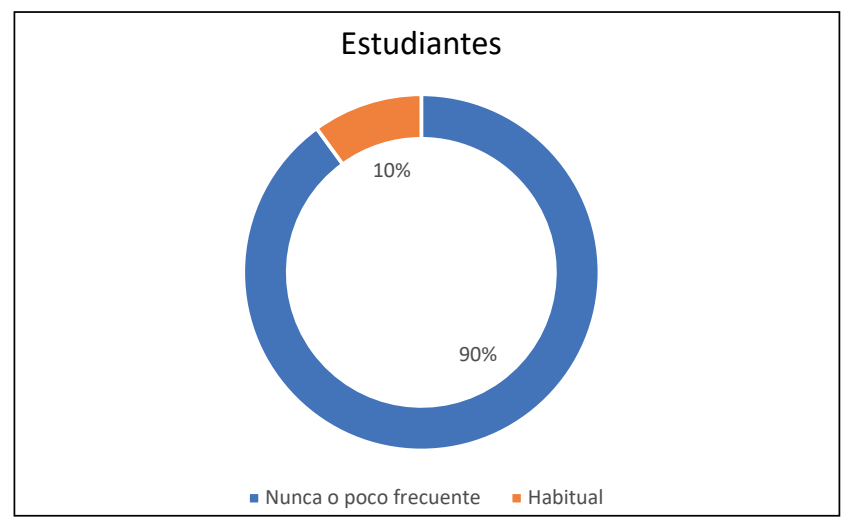

Figura 2. Trabajo en equipo previo al curso de estudiantes. 


\section{Trabajo en equipo durante al curso}

Al tener que utilizar el trabajo en equipo se aprecia un radical cambio en la aceptación de esta forma de trabajo. Los estudiantes que previamente habían trabajado en equipo en una menor medida que los profesores han podido poner en marcha este método de trabajo de una manera mucho más natural que los profesores. La aceptación del trabajo en equipo por parte de los estudiantes coincide con un estudio realizado por Francis y Hoefel (2017) en el cual se detectó que la generación Z es mucho más comunicativa (Ilamados communicaholics) y abierta al diálogo que generaciones anteriores. Igualmente,

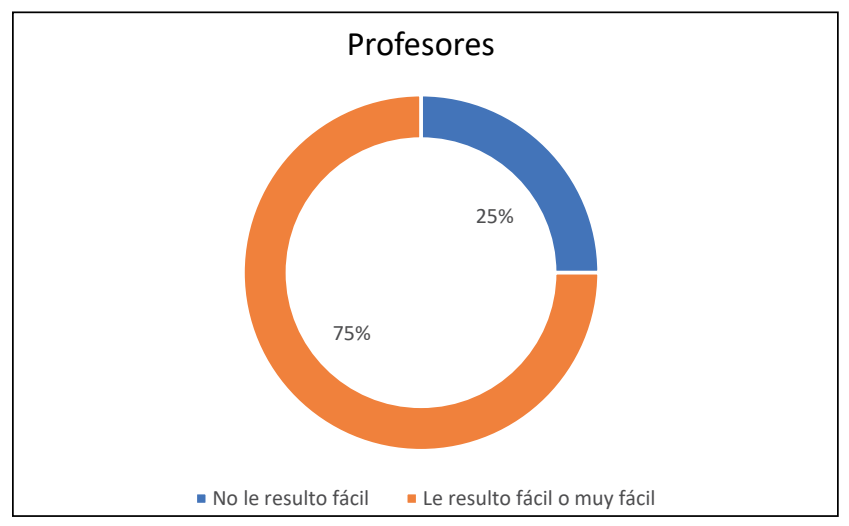

Figura 3. Trabajo en equipo durante el curso de profesores.

\section{Colaboración con el aprendizaje de sus compañeros}

Los estudiantes fueron más colaborativos con otros integrantes del equipo en aquellas tareas que a sus compañeros le resultaban más difíciles de cumplir, comparados con los profesores que les estos resultados concuerdan con un interesante estudio realizado en cuatro universidades españolas para identificar la percepción de los alumnos sobre el trabajo en grupo, en el que se concluye "que los alumnos, independientemente de su universidad de procedencia, muestran altas percepciones para trabajar en grupo y de manera colaborativa" (Cabero y Marín, 2013, p.171). Lo anterior evidencia que este tipo de trabajo además de ser útil para tomar decisiones teniendo en cuenta la diversidad de perspectivas, es eficaz para el desarrollo del producto o trabajo a realizar y tiene beneficios para el aprendizaje.

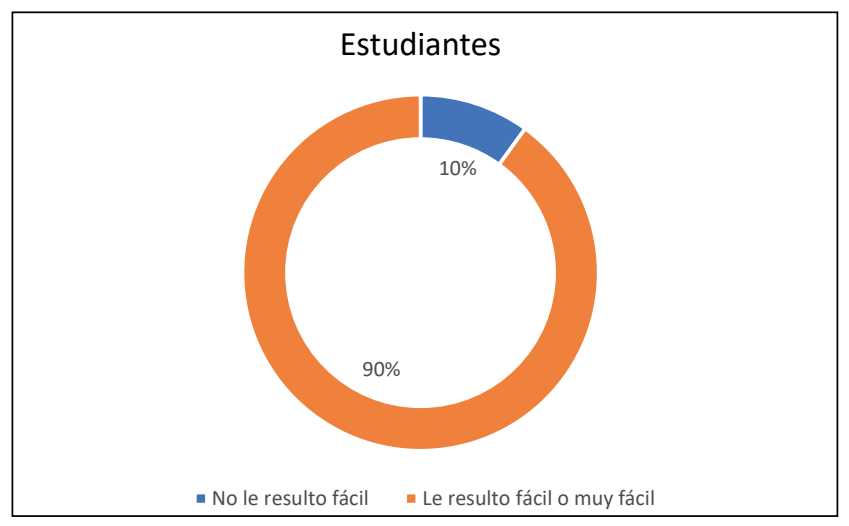

Figura 4. Trabajo en equipo durante el curso de estudiantes.

ha resultado más difícil colaborar con sus compañeros. Según el estudio de Dell Technologies (2018), el 77 \% de los estudiantes de la generación $Z$ desean ser mentores tecnológicos para otros de su organización, lo cual se ve reflejado en la alta participación que los mismos tienen en colaborar con el aprendizaje de sus pares. 


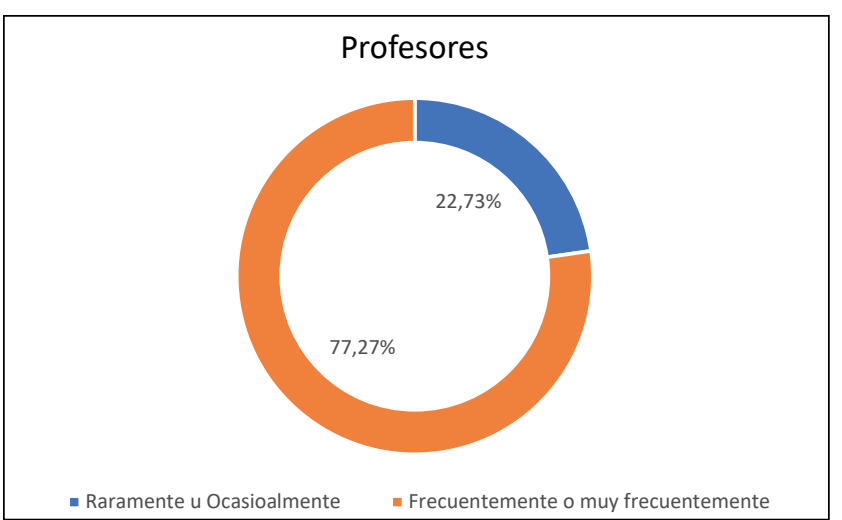

Figura 5. Colaboración entre profesores con el aprendizaje de sus compañeros.

\section{Sensación al evaluar a sus compañeros}

Según Pastor (2005), los estudiantes al tener que evaluar a sus compañeros muestran interés y lo realizan de manera correcta. Lo cual coincide con las expresiones de los estudiantes que demuestran que se sintieron más cómodos al evaluar a sus

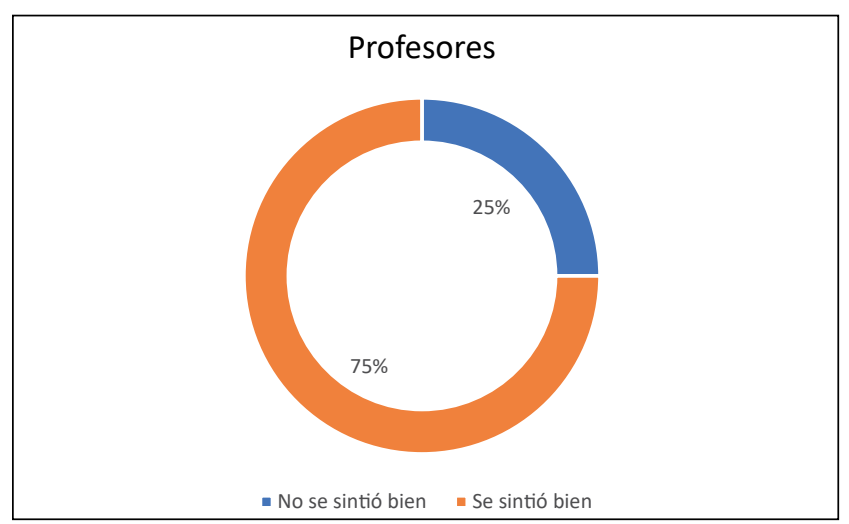

Figura 7. Sensación al evaluar a sus compañeros profesores.

\section{Uso de TIC previo al curso}

Como se muestra en la figura 9 , el $68 \%$ de los profesores usaban TIC en los procesos de enseñanza con sus alumnos de manera habitual. La figura 11 muestra que al 91 \% le resultó fácil la utilización de TIC durante el transcurso del curso de formación. Los estudiantes realizaban un uso cercano al de los profesores de las TIC previo al curso, siendo

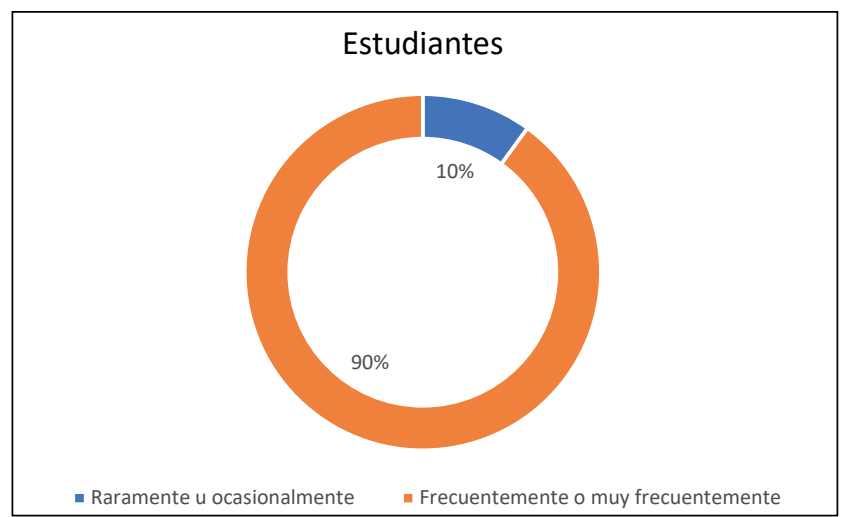

Figura 6. Colaboración entre estudiantes con el aprendizaje de sus compañeros.

compañeros que los profesores al hacerlo con sus homólogos. Es importante destacar que los profesores practican la evaluación de manera constante, mientras que los alumnos no estaban habituados a evaluar a sus compañeros; aún así, estos últimos se sintieron mejor realizando las evaluaciones.

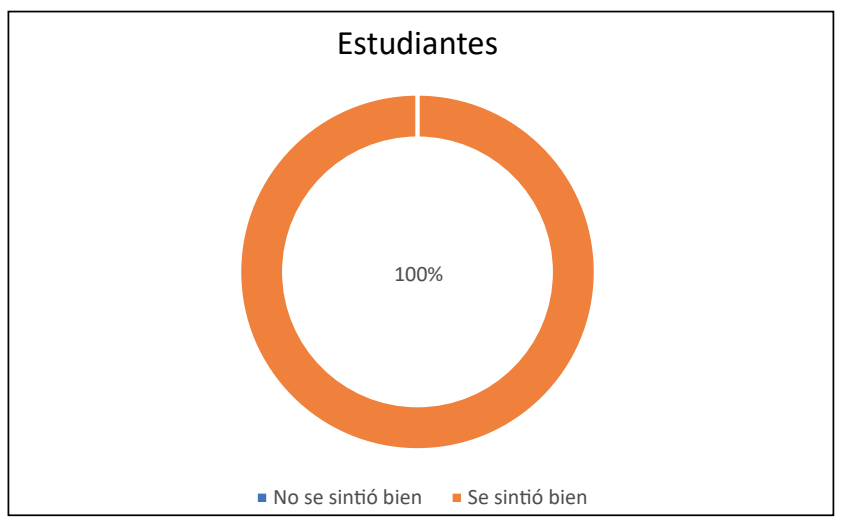

Figura 8. Sensación al evaluar a sus compañeros estudiantes.

del $70 \%$ (figura 10) del uso de TIC en estudiantes versus $68.18 \%$ (figura 9) en profesores. La figura 12 pone de manifiesto que al $20 \%$ de los estudiantes no le resultó fácil el uso de las tecnologías durante el curso, mientras que solamente el $9 \%$ (figura 11) de los profesores tuvieron inconvenientes con el uso de las TIC. Tanto profesores como estudiantes realizaban un uso similar, en términos porcentuales, de las TIC para el aprendizaje-enseñanza. 


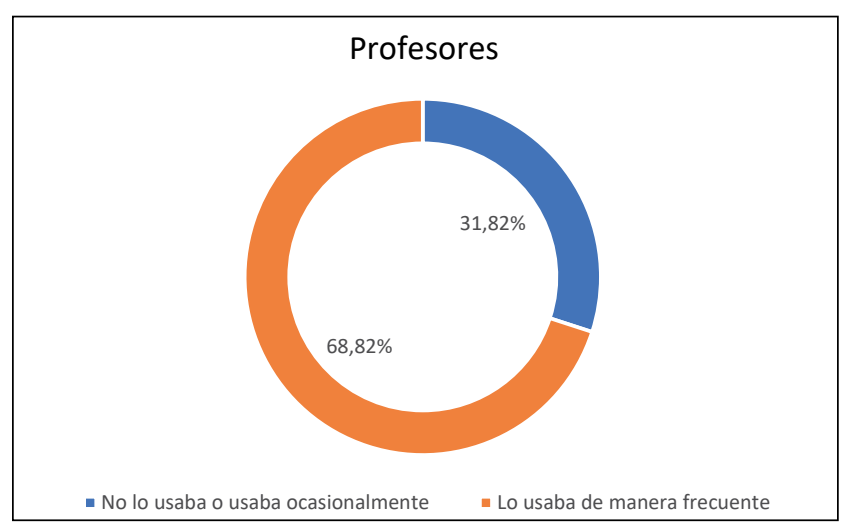

Figura 9. Uso de TIC de los profesores previo al curso.

\section{Uso de TIC durante el curso}

Durante el curso se establecieron diversos ejercicios y prácticas en los cuales era indispensable el uso de las TIC como fuente de conocimiento. Algunos de los estudiantes argentinos tenían conocimientos muy básicos de tecnologías y durante el curso tuvieron que utilizar muchas herramientas tecnológicas que antes no utilizaban. La gran mayoría manifestó que aprendieron a utilizar herramientas de soporte tecnológico en sus trabajos, mientras que los que no habían tenido trabajos previos al curso tuvieron su primer acercamiento a estas tecnologías durante el curso. Este acercamiento a las tecnologías les enfrentó a

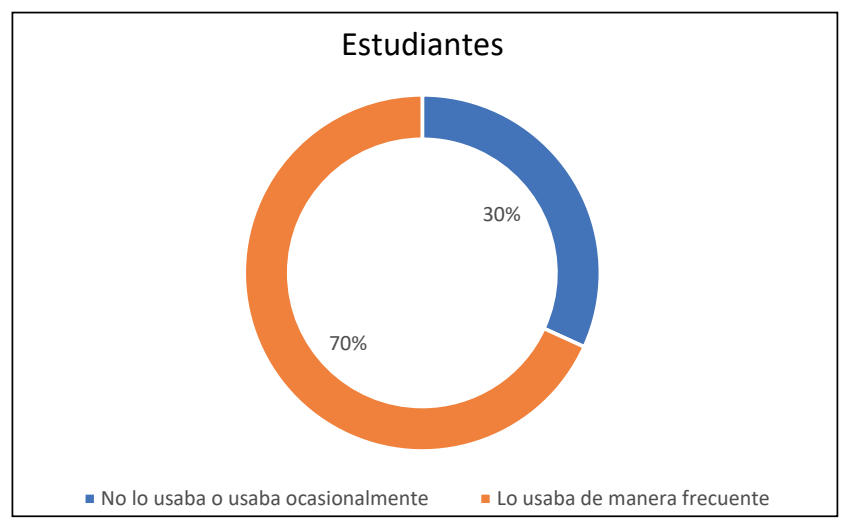

Figura 10. Uso de TIC de los estudiantes previo al curso.

situaciones en las cuales sus modelos mentales no funcionaban, teniendo que buscar nuevos para poder aplicar. Este concepto es desarrollado por Bain (2007) bajo el concepto de "El conocimiento es construido, no recibido". En el caso de los docentes colombianos, en su mayoría mostraron pocas habilidades para búsquedas sencillas y básicas de documentos e información durante los ejercicios prácticos del curso, mostrando algunas veces resistencia al descubrir que el facilitador no es siempre la única fuente de información, como bien lo advierte Salinas (2004) cuando recalca la necesidad de un cambio de cultura y de funciones de profesores y estudiantes en ambientes ricos en TIC.

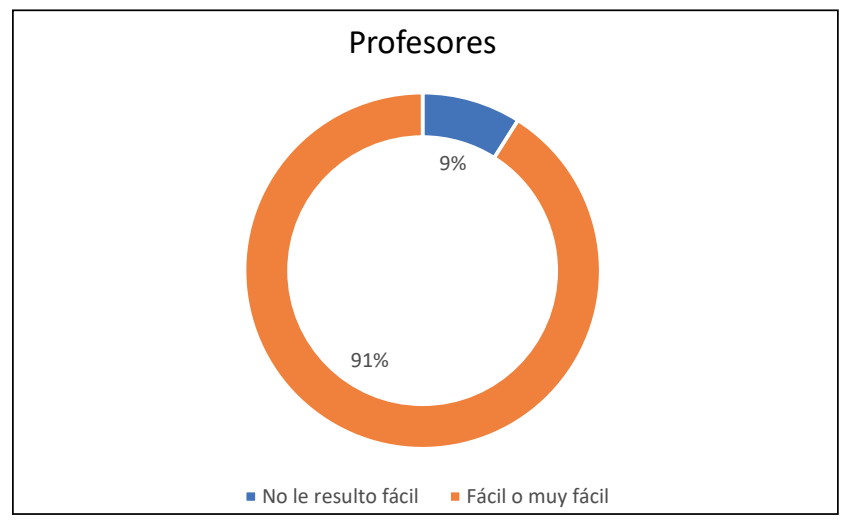

Figura 11. Uso de TIC de profesores durante el curso.

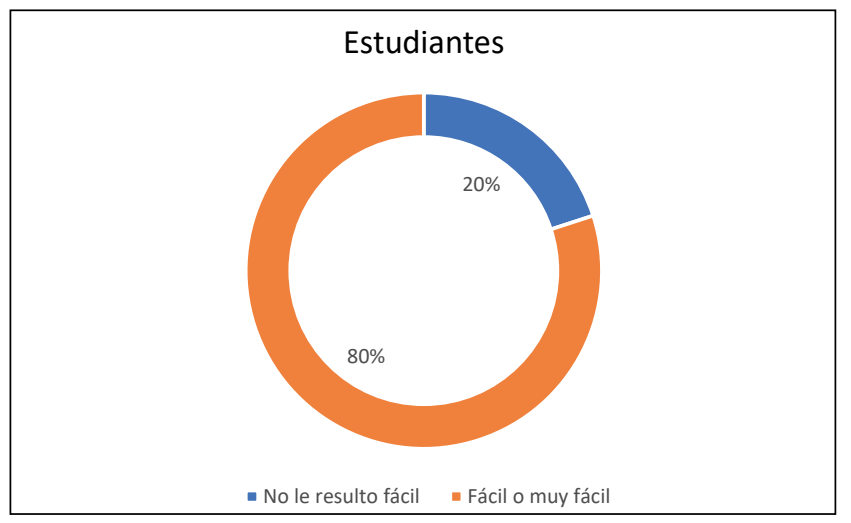

Figura 12. Uso de TIC de estudiantes durante el curso. 


\section{Tecnologías utilizadas para comunicarse con sus compañeros}

La figura 13 pone en manifiesto que los profesores siguen prefiriendo el correo electrónico como método de comunicación primario con sus compañeros, mientras que los alumnos (figura 14) prefieren otras tecnologías de comunicación. Según Ramírez (2017), los docentes se encuentran en una etapa exploradora y su comunicación sigue siendo a través de correo electrónico como única vía. Al consultarle a los estudiantes de

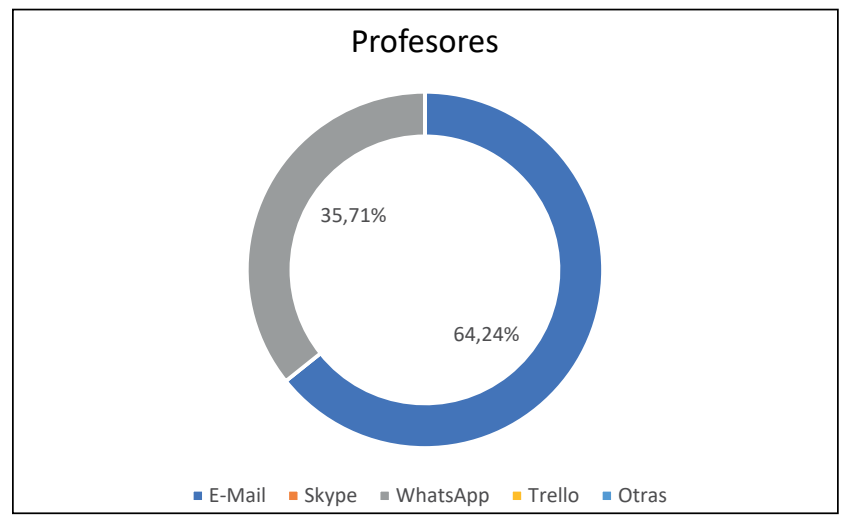

Figura 13. Tecnologías utilizadas por los profesores

Fuentes de información utilizadas durante el curso

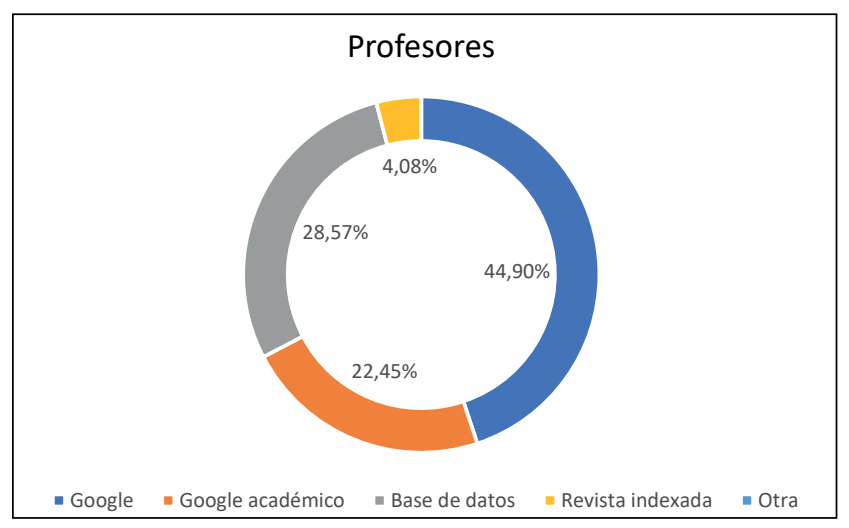

Figura 15. Fuentes de información utilizadas por profesores durante el curso.
Argentina sobre las otras tecnologías utilizadas, mencionaron principalmente Slack (herramienta similar a WhatsApp, pero con una orientación a la comunicación empresarial/formal). Tanto para profesores como alumnos WhatsApp es el segundo más elegido para intercambiar información entre sus compañeros. Se puede apreciar que el correo electrónico es una herramienta muy utilizada por los profesores para la comunicación con otros miembros del equipo, pero la misma no es utilizada por los estudiantes, prefiriendo otros métodos de comunicación entre pares.

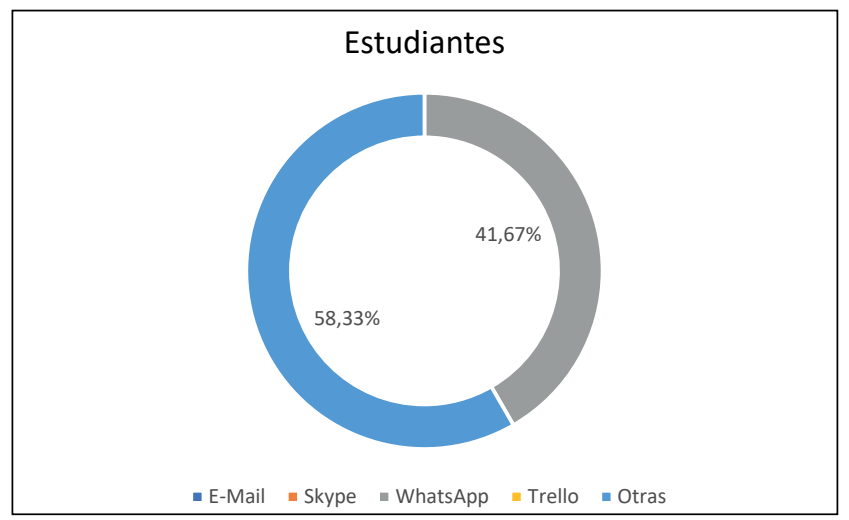

Figura 14. Tecnologías utilizadas por los estudiantes para comunicarse con sus compañeros.

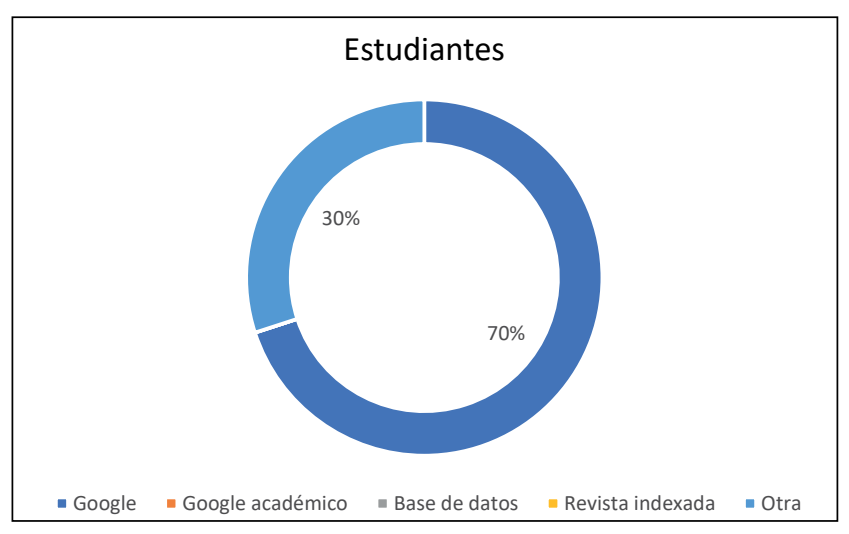

Figura 16. Fuentes de información utilizadas por los estudiantes durante el curso. 
La principal fuente de información que utilizan los profesores y estudiantes es Google (figura 15 y figura 16), los profesores utilizándola como fuente principal en un $44.90 \%$ (figura 15) mientras que los alumnos en un $30 \%$ (figura 16). Los profesores son los que utilizan medios más especializados como Google Académico, bases de datos y revistas indexadas. Los estudiantes no utilizaron los medios Ilamados formales, prefiriendo utilizar sitios web del estilo foro, libros digitales y video tutoriales.

\section{Conclusiones}

El aprendizaje colaborativo y la aplicación de las TIC al proceso de aprendizaje son dos de las competencias más mencionadas en las propuestas sobre el perfil global del estudiante y del profesor. Un perfil global se compone de capacidades o competencias disciplinares globales y competencias o habilidades transversales globales.

El proyecto de innovación "Resistencias en la implementación de nuevas didácticas para el desarrollo de competencias globales" se realizó por los autores del artículo, con el propósito de desarrollar durante unos cursos de formación esas dos competencias en dos grupos de estudiantes y dos grupos de profesores de la educación superior.

Los resultados del proyecto de innovación en los grupos de docentes y estudiantes muestran que para los primeros el uso de TIC y el aprendizaje colaborativo resulta más difícil de aplicar, pero cuando deben lo hacen sin mayores resistencias.

Antes de la participación en los cursos los docentes y los estudiantes practicaban el aprendizaje colaborativo de manera casual.

En el ejercicio práctico, formal, planeado y sistemático de técnicas de aprendizaje colaborativo los estudiantes universitarios se mostraron más cómodos y abiertos que los docentes universitarios.

Definitivamente en el proyecto de innovación se denota que a los estudiantes universitarios les es más natural trabajar de manera colaborativa y aplicar las TIC de mayor complejidad, lo que plantea un reto para cerrar esta brecha generacional de competencias del perfil global. Hay que señalar que el proyecto de innovación aporta dos instrumentos útiles para identificar qué tanto aceptan los estudiantes las técnicas de aprendizaje colaborativo y TIC.

Cuando se busca un perfil global del estudiante es esencial que el docente se mantenga actualizado y pueda utilizar herramientas modernas y de uso común en los ambientes laborales. Los docentes, en su mayoría, nacieron y crecieron antes del auge de las tecnologías que son aplicadas actualmente, por lo que el reto es mayor, ya que no solo deben aprender las nuevas tecnologías, sino que también deben poder incorporarlas en sus propios aprendizajes. El desafío se encuentra en cómo mantener a los profesores, y estudiantes, interesados en la constante incorporación de nuevas habilidades y competencias para poder lograr una educación de mayor calidad y con mejores profesionales.

\section{Referencias}

Aponte, C., Botero, L. H., Aristizábal, M., Arroyave, J. J., Cuevas, M. P. y Muñoz, S. (2014). Currículo internacional: dimensiones para la formación del estudiante. Colombia: Ediciones Universidad de Medellín.

Aponte, C. (2018). Competencias del perfil global: Caja de herramientas para la Internacionalización del Currículo. Medellín: Instituto Tecnológico Metropolitano. Recuperado de https://www. itm.edu.co/dependencias/direccion-de-cooperacion-y-relaciones-internacionales-5/ internacionalizacion-del-curriculo/caja-de-herramientas/

Aponte, C. y Jordan, R. (2017). Formación del ingeniero global. En II Congreso Internacional y V Nacional de Ingeniería Electromecánica, Universidad Pedagógica y Tecnológica de Colombia.

Aponte, C., Jordan, R. y Agi, K. (2017). International Profile of a Global Engineer. En World 
Engineering Education Forum (WEEF). Recuperado de http://c3educationlab.com/wp-content/ uploads/2018/12/1-WEEF-2017-Aponte-JordanAgi-International-Profile-of-a-Global-Engineer.pdf

Bain, K. (2007). Lo que hacen los mejores profesores de universidad. Valencia: Universitat de Valencia.

Begué, A., L., Bedoya, M. M., Calle, J. M., Lizón, L. A., Rivera J. G. y Rojas, C. A. (2012). Orientaciones pedagógicas. Medellín: Instituto Tecnológico Metropolitano.

Benvenuto, A. (2003). Las tecnologías de información y comunicación (TIC) en la docencia universitaria. Theoria, 12(1), 109-118.

Barroso Ramos, C. (2007). La incidencia de las TIC en el fortalecimiento de hábitos y competencias para el estudio. Edutec, Revista Electrónica de Tecnología Educativa, 23. https://doi.org/10.21556/ edutec.2007.23.497

Cabero, J. y Marín, V. (2013). Posibilidades educativas de las redes sociales y el trabajo en grupo. Percepciones de los alumnos universitarios. Comunicar. Revista Científica de Educomunicación, 11(42), 165-172.

Dell Technologies (2018). Gen Z: the future has arrived. Recuperado de https://www.delltechnologies.com/content/dam/digitalassets/active/en/ unauth/sales-documents/solutions/gen-z-the-future-has-arrived-executive-summary.pdf

Felder, R. y Brent, R. (2003). Designing and Teaching Courses to Satisfy the ABET Engineering Criteria. Journal of Engineering Education, 92(1), 7-25. https://doi.org/10.1002/j.2168-9830.2003. tb00734.x

Francis T. y Hoefel F. (2017). 'True Gen': Generation $Z$ and its implications for companies. Recuperado de https://www.mckinsey. com/industries/consumer-packaged-goods/ our-insights/true-gen-generation-z-and-its-implications-for-companies

Iste (2016). Estándares Iste en TIC para estudiantes. Recuperado de: http://eduteka.icesi.edu.co/articulos/ estandares-iste-estudiantes-2016

Lillo, F. (2013). Aprendizaje Colaborativo en la Formación Universitaria de pregrado. Revista de Psicología, 2(4), 102-142.

Maldonado, M. (2007). El trabajo colaborativo en el aula universitaria. Revista de Educación Laurus, 13(23), 263-278.

Pastor, V., González, M. y Barba, J. (2005). La participación del alumnado en la evaluación: La autoevaluación, la coevaluación y la evaluación compartida. Tandem: Didáctica de la Educación Física, 17, 21-37.

OCDE (2018). Preparing our youth for an inclusive and sustainable world. The OECD PISA global competence framework. Recuperado de https://www. oecd.org/education/Global-competency-for-an-inclusive-world.pdf

OCDE (1998). Making the curriculum work. París: OCDE. https://doi.org/10.1787/9789264163829-en

OCDE (s. f. ). Working Paper 21st Century Skills and Competences for New Millennium Learners in OECD Countries, EDU Working paper, 41.

Ramírez, M. A. (2017). Impacto en las prácticas de aula de la formación docente en TIC para el desarrollo profesional docente en la Institución de Educación Superior-ITFIP. Recuperado de https:// reposital.cuaed.unam.mx:8443/xmlui/bitstream/ handle/20.500.12579/5064/VE17.1120__366012b5.pdf? sequence $=1$ \&isAllowed $=y$

Salina, J. (2004). Innovación docente y uso de las TIC en la enseñanza. Revista de Universidad y Sociedad del Conocimiento, 1(1), 1-16.

\section{(c) $(1) \Theta$}

